\title{
PHOTODISSOCIATION AND LARGE INTERNUCLEAR DISTANCE DYNAMICS
}

\author{
M. GLASS-MAUJEAN \\ Laboratoire de Spectroscopie Hertzienne de l'ENS† Université \\ P. et M. Curie, 4 pl Jussieu 75252 Paris, cedex 05 and LURE $\ddagger$ \\ Bât 209D Campus d'Orsay, 94405 Orsay cedex
}

\section{J. A. BESWICK}

LURE $\ddagger$ Bât 209D Campus d'Orsay, 94405 Orsay cedex

\section{H. FROHLICH}

Laboratoire des Collisions Atomiques et Moleculaires, $\dagger$ Bât 351, Université Paris-Sud, 94405 Orsay cedex and LURE Bât 209D, Campus d'Orsay, 94405 Orsay cedex

\begin{abstract}
The determination of the state of the excited photofragments gives informations on couplings occurring at large internuclear distance. The $\mathrm{H}(1 \mathrm{~s})+\mathrm{H}(n=2)$ photodissociation of $\mathrm{H}_{2}$ is well explained with a transition probability between the $\mathrm{B}$ and $\mathrm{B}^{\prime}$ continua occurring at $r \approx 15 a_{0}$. The $\mathrm{H}(1 \mathrm{~s})+\mathrm{H}(n=3)$ photodissociation of $\mathrm{H}_{2}$ presents unsolved questions.
\end{abstract}

KEY WORDS: Photodissociation, long-range couplings, molecular hydrogen.

\section{INTRODUCTION}

Photodissociation is often presented as a half collision but most of the time the problems studied by each approach are quite different.

Photodissociation is generally studied from cross section measurements, then the main facts discussed are the structures and the predissociation mechanisms which involve couplings to a continuum at short or intermediate distances. These couplings barely account in collision problems. In which conditions a photodissociation may be compared to a collision? Let us say when a photodissociation is sensitive to interactions at large internuclear distances.

Consider a photodissociation yielding to fragments with energy levels nearly degenerated (an $\mathrm{H}^{*}$ excited atom or an atom with a spin-orbit splitting). The final state of the fragment will be a superposition of states and this superposition will

$\dagger$ Unités Associées au CNRS.

‡ Laboratoire du CNRS, CEA et MEN. 
depend on the energy of dissociation. To determine the dissociation final state or to look to the collision spin-orbit transfer are two techniques to study the same problem.

\section{THE $\mathrm{H}(n=2)+\mathrm{H}(1 s)$ PHOTODISSOCIATION OF $\mathrm{H}_{2}$}

The photodissociation of molecular hydrogen is typically such of these problems. It leads to one excited fragment with orbital degenerated states. The main features of the first optically allowed photodissociation of $\mathrm{H}_{2}$ are due to the predissociation of the $\mathrm{D}\left(3 p \pi{ }^{1} \Pi_{u}\right)$ state. ${ }^{1}$ Such predissociation is induced by the $\mathrm{B}^{\prime}\left(3 p \sigma^{1} \Sigma_{u}^{+}\right)$state correlated to $\mathrm{H}(2 s)+\mathrm{H}(1 s)$ fragments. In fact only $60 \%$ of the excited atoms were measured in the $2 s$ state. $^{2}$

This was explained by Komarov and Ostrovsky: ${ }^{3}$ at large internuclear distance $\left(r_{0} \approx 15 a_{0}\right)$ and the $\mathrm{B}^{\prime}$ state correlated to $\mathrm{H}(2 s)+\mathrm{H}(1 s)$ and the $\mathrm{B}\left(2 p \sigma^{1} \Sigma_{u}^{+}\right)$state correlated to $\mathrm{H}(2 p)+\mathrm{H}(1 s)$ are both coupled to the $\mathrm{H}^{+}+\mathrm{H}^{-}$ion pair state which induces a non-adiabatic transition between them. Then a definite molecular state may well yield to two different dissociated states.

On the opposite if we consider the direct dissociation, both B and $\mathrm{B}^{\prime}$ continua can be excited. Two dissociative paths will lead to the same final state. The $\mathrm{H}(2 s)+\mathrm{H}(1 s)$ final state, for distance, can be obtained by photoabsorption into the $\mathrm{B}^{\prime}$ state followed by adiabatic dissociation or by photoabsorption into the $\mathrm{B}$ state followed by non-adiabatic transition into the $\mathrm{B}^{\prime}$ state and similarly for the $\mathrm{H}(2 p)+\mathrm{H}(1 s)$ channel.

A complete quantal description of the system has been made. ${ }^{4}$ It has been shown that the quantum results can be reproduced by a collision perturbative model, in which the partial photodissociation cross sections are equal to:

$$
\begin{aligned}
& \sigma_{\mathrm{P}}=\sigma_{\mathrm{B}}, P+\sigma_{\mathrm{B}}(1-P)-2\left[\sigma_{\mathrm{B}^{\prime}}, \sigma_{\mathrm{B}} P(1-P)\right]^{1 / 2} \cos \left(\delta_{\mathrm{B}},-\delta_{\mathrm{B}}\right)+\sigma_{\mathrm{C}} \\
& \sigma_{S}=\sigma_{\mathrm{B}},(1-P)+\sigma_{\mathrm{B}} P+2\left[\sigma_{\mathrm{B}^{\prime}}, \sigma_{\mathrm{B}} P(1-P)\right]^{1 / 2} \cos \left(\delta_{\mathrm{B}},-\delta_{\mathrm{B}}\right)
\end{aligned}
$$

where $\sigma_{\mathrm{C}}, \sigma_{\mathrm{B}^{\prime}}$ and $\sigma_{\mathrm{B}}$ represent the photoabsorption cross sections into the $C\left(2 \mathrm{p} \pi^{1} \Pi_{u}\right), \mathrm{B}^{\prime} \mathrm{B}$ respectively, $\delta_{\mathrm{B}}$ and $\delta_{\mathrm{B}^{\prime}}$, represent the phases of the continuum vibrational wavefunctions and $P$ the non-adiabatic transition probability to jump from one state to the other during the half collision. ${ }^{4}$ The phase difference is expected to vary with excess energy. For squared potential wells it varies like $(\Delta E)^{1 / 2}$ giving an oscillatory behaviour to the partial cross section. This has been observed experimentally on the branching ratio $\sigma_{P} / \sigma_{S}{ }^{5}$

Dispersed synchrotron radiation was used to photodissociate $\mathrm{H}_{2}$ at low pressure in a differentially pumped cell. The $\mathrm{H}(2 p)$ excited fragments have a short radiative lifetime and their $\mathrm{Ly}_{\alpha}$ fluorescence is easily detected. The $\mathrm{H}(2 s)$ fragments are metastable. An applied electric field is used to induce mixing between the $2 s$ and $2 p$ states, allowing $\mathrm{Ly}_{\alpha}$ emission. Extrapolation to zero pressure has to be used to determine the branching ratio $\sigma_{P} / \sigma_{S}$. This technique had already been applied more than ten years ago to a $\mathrm{D}$ predissociation peak. ${ }^{2}$ 
The extrapolated ratio between the detected fluorescence with the electric field off and that with it on is:

$$
R=\alpha \sigma_{P} /\left(\alpha \sigma_{P}+\beta \sigma_{S}\right)
$$

where $\alpha$ and $\beta$ are corrective factors for the anisotropy of the fluorescence. $\beta=0.903$ and $\alpha$ is expected to vary between 0.79 and 0.90 depending on the excess energy in our experimental conditions. ${ }^{6}$

The $R$ ratio has been measured at six different energies in the direct dissociation continuum and at the summit of a predissociation peak. On the peak the measured value for $\alpha \sigma_{P} / \sigma_{S}$ is $0.71 \pm 0.09$ in agreement with the previous value after taking into account the detection symmetry. ${ }^{2}$ In Figure 1 is presented a comparison between the experimental values for $\alpha \sigma_{P} / \sigma_{S}$ and (a) the theoretical predictions in the adiabatic limit, (b) the calculations from (Eq. 1) giving to $P$ the value $0.43 \pm 0.02$ deduced from the measured branching ratio on the resonance peak where $\sigma_{\mathrm{B}^{\prime}}, \gg \sigma_{\mathrm{B}}, \sigma_{\mathrm{C}}$. In such conditions $\alpha$ was calculated to be equal to 0.9 . The potential curves used to calculate the cross sections and the vibrational phases are from Kolos et al..$^{7-10}$ The points calculated from the empirical determination of $P$ are in very good agreement with the experiment. That value of $P$ is very near that of Komarov and Ostrovsky ${ }^{3}$ obtained by a semi-classical approach.

In conclusion of this part we will say that the branching ratio $\mathrm{H}(2 p)$ to $\mathrm{H}(2 s)$ in the photodissociation of $\mathrm{H}_{2}$ is observed to vary strongly with the excess energy. This branching ratio is well encountered by a half collision description in which a

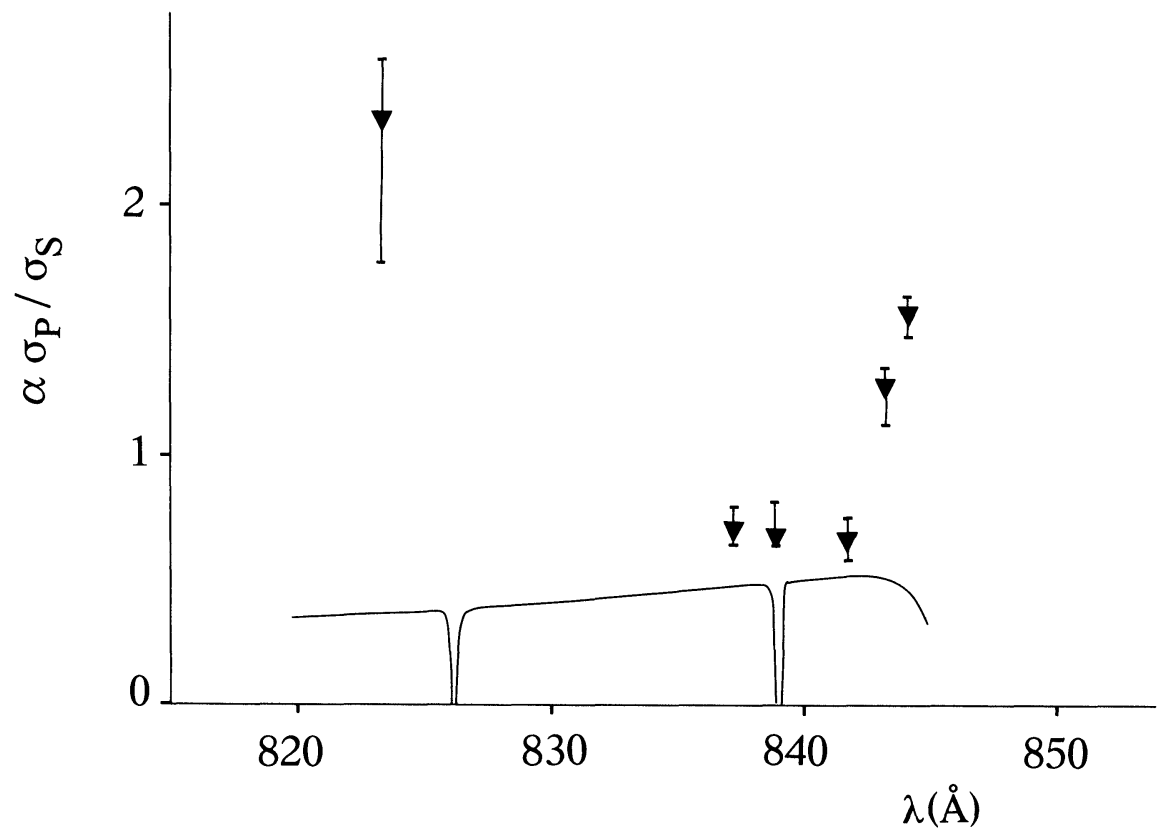

Figure 1 Comparison between the experimental values for $\alpha \sigma_{P} / \sigma_{S}$ (with the error bars) and (a) the theoretical predictions in the adiabatic limit (full line), (b) the calculations with $P=0.43 \pm 0.2$ (triangles). 
transition between two paths may occur at large internuclear distance. The transition probability had been determined. What occurs at the second allowed photodissociation limit?

\section{THE $\mathrm{H}(n=3)+\mathrm{H}(1 s)$ PHOTODISSOCIATION OF $\mathrm{H}_{2}$}

For incident wavelengths shorter than $750 \AA$, dissociation into $\mathrm{H}(n=3)+\mathrm{H}(1 s)$ may occur. Such dissociation can be detected monitoring the Balmer $\alpha$ emission $(\mathrm{H}(n=3) \mathrm{H}(n=2)$ at $6560 \AA)$. Its excitation spectrum has not yet been assigned (Figure 2). We may presume that the predissociated levels are high vibrational levels of the lower Rydberg series $4 p \pi, 5 p \pi^{1} \Pi_{u}$ or $5 p \sigma^{1} \Sigma_{u}$. In $\mathrm{H}_{2}$ for dissociation to compete successfully against ionization, ionization has to occur with a large vibrational change $(\Delta v \gg 1)$ as vibrational interaction is the most efficient mechanism. ${ }^{11}$ By a rapid extension from the situation of the $\mathrm{H}(1 s)+\mathrm{H}(n=2)$ dissociation, where all the predissociations occur through the $\mathrm{B}^{\prime}$ continuum,${ }^{12}$ one may expect that the $\mathrm{H}(n=3)+\mathrm{H}(1 s)$ predissociations proceed from the $\mathrm{B}^{\prime \prime}{ }^{1} \Sigma_{u}^{+}$continuum.

Similarily to the $\mathrm{H}(n=2)+\mathrm{H}(1 s)$ case, at very large distance $\left(R \approx 35 a_{0}\right)$ the degenerated ${ }^{1} \Sigma_{u}^{+} \mathrm{H}(n=3)+\mathrm{H}(1 s)$ diabatic curves are coupled to the ion pair state inducing some transfer probabilities between the $n=3$ states. ${ }^{13}$ Then on a predissociation peak, coming from the $\mathrm{B}^{\prime \prime}$ state correlated to $\mathrm{H}(3 p)+\mathrm{H}(1 s)$, some branching ratio $3 \mathrm{~S}: 3 \mathrm{P}: 3 \mathrm{D}$ is expected, independent of the peak. We did observe such branching ratios.

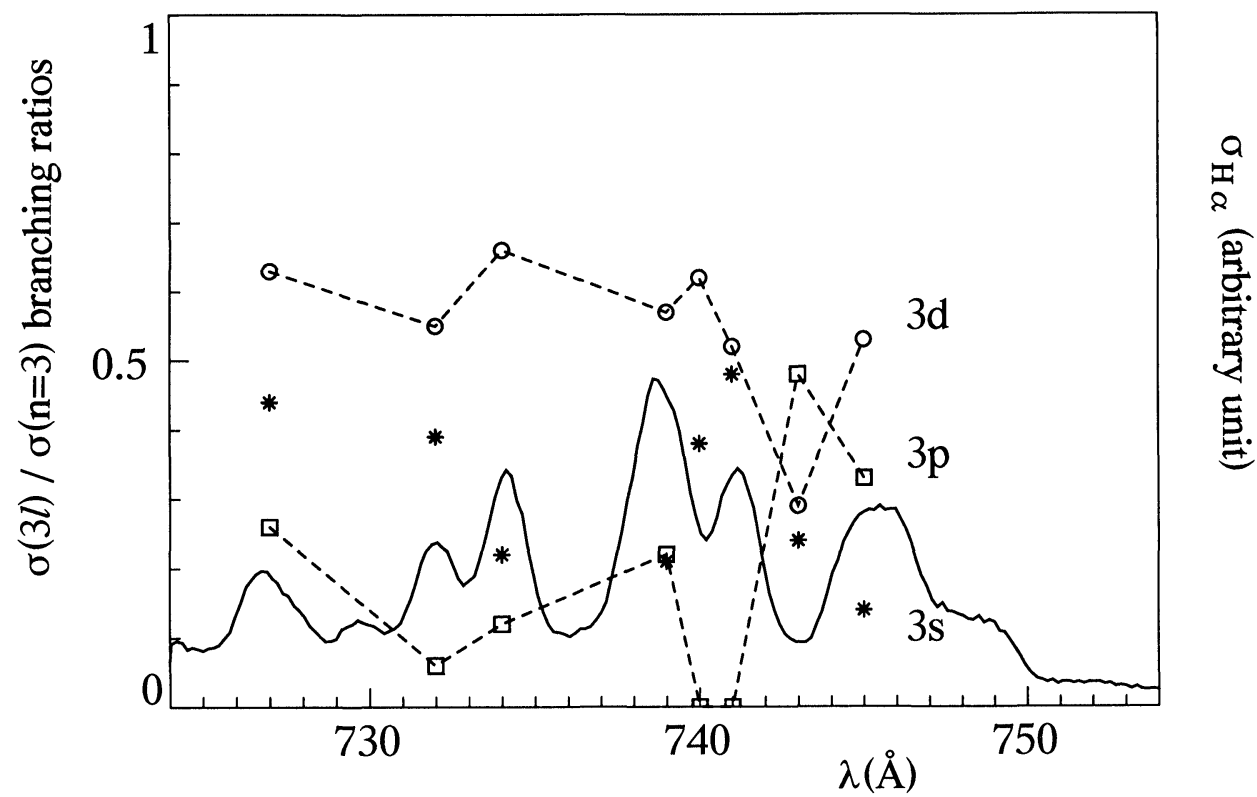

Figure 2 Balmer $\alpha$ excitation spectrum, and $\sigma(3 l)(/ \sigma(n=3)$ measured ratios. 


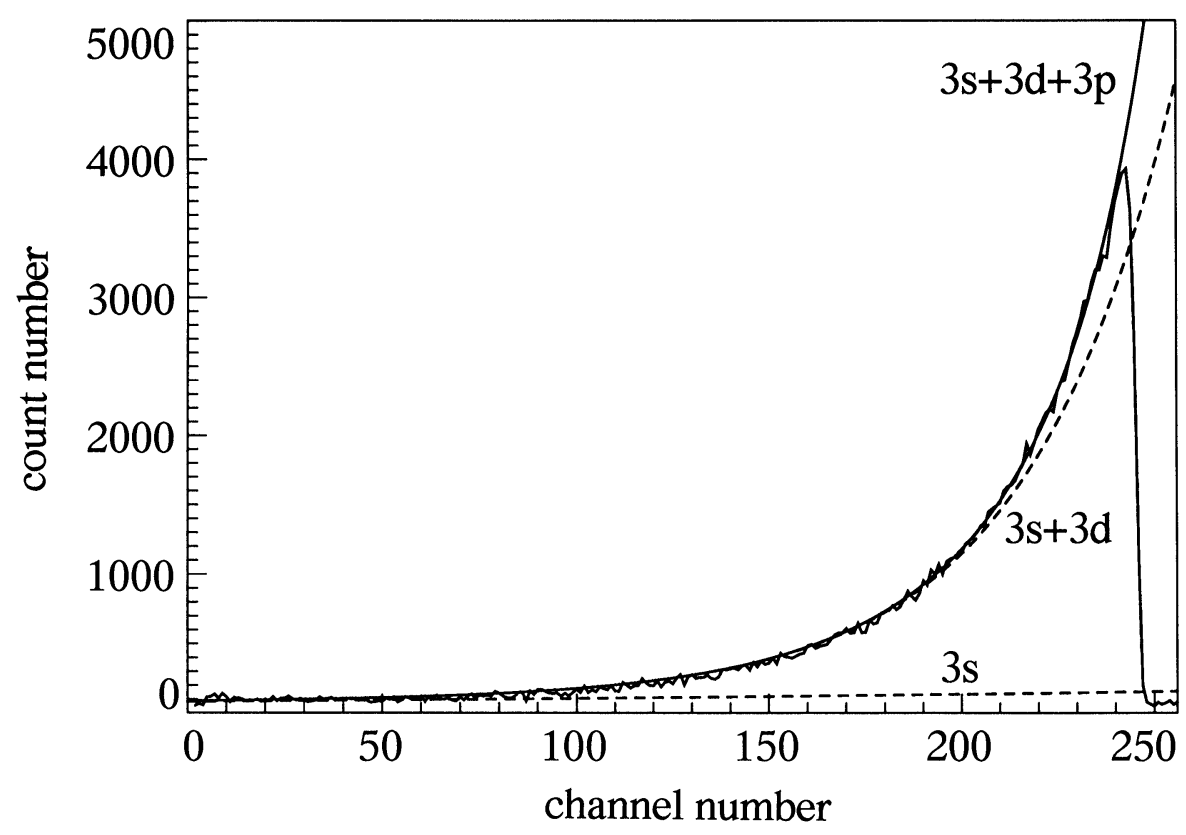

Figure 3 Balmer $\alpha$ time analysis at $\lambda=745 \AA$, and the corresponding fit.

Balmer $\alpha$ fluorescence was detected perpendicularly to the incident synchrotron light beam, through a light pipe, selected by a colored filter, by a refrigerated photomultiplier. We took advantage of the pulsed character of the synchrotron radiation to perform a time analysis of the $\mathrm{H}_{\alpha}$ fluorescence. The predissociations occur in a time very short compared to atomic decay times, then the fluorescence decay is just the superposition of the $\mathrm{H}(3 s, 3 p$ and $3 d)$ atomic exponential decays. The atomic lifetimes are quite different: $158,5.3$ and $15.5 \mathrm{~ns}$ respectively and can be easily distinguished. ${ }^{14}$ When operating with two positron packets, the delay between two incident light-pulses is of $115 \mathrm{~ns}$, the analysis time is of $100 \mathrm{~ns}$ for 256 channels.

We determined the branching ratios of six predissociation peaks and one vale. For each incident wavelength the decay curve was analysed as a sum of exponentials of definite width corresponding to the theoretical lifetimes. The populations have been deduced from the integration of the related decay over the time running between two consecutive incident pulses, taking into account that all the $3 s$ and $3 d$ states radiate through Balmer emission, whilst only $15 \%$ of the $3 p$ do. Figure 3 displays such a fit. The results are summarized on Figure 2.

Obviously, the branching ratios show strong variations even for the predissociation peaks. The assumption described above is too simple. The $\mathrm{B}^{\prime \prime}$ continuum is probably not the only one to play a role, the $\mathrm{D}^{1} \Pi_{u}$ continuum may be efficient too. The D state is correlated to $\mathrm{H}(3 p)+\mathrm{H}(1 s)$. Once again, the system is dissociating coming from a $p$ configuration, but as it is of a $\Pi$ symmetry, no mixing with the $3 \mathrm{~s}$ 
configuration may occur at large distance. That may explain some variations observed on the branching ratios.

In conclusion, the study of the branching ratios shows the highly complexity of the large distance couplings in the case of degenerated fragment states, more theoretical work is needed to understand the experimental results.

\section{References}

1. M. Glass-Maujean, J. Breton and P. M. Guyon, Chem. Phys. Lett. 63, 591 (1979).

2. J. E. Mentall and P. M. Guyon, J. Chem. Phys. 67, 3845 (1977).

3. I. V. Komarov and V. N. Ostrovsky, J. Phys. B12, 2485 (1979).

4. J. A. Beswick and M. Glass-Maujean, Phys. Rev. A35, 3339 (1987).

5. M. Glass-Maujean, H. Frohlich and J. A. Beswick, Phys. Rev. Lett. 61, 157 (1988).

6. M. Glass-Maujean and J. A. Beswick, Phys. Rev. A38, 5660 (1988).

7. W. Kolos and L. Wolniewicz, Can. J. Phy. 53, 2189 (1975).

8. W. Kolos and J. Rychlewski, J. Mol. Spectrsc. 62, 109 (1976).

9. W. Kolos, J. Mol. Spectrosc. 62, 429 (1976).

10. W. Kolos and L. Wolniewicz, J. Mol. Spectrosc. 54, 303 (1975).

11. P. M. Dehmer and W. A. Chupka, J. Chem. Phys. 65, 2243 (1976).

12. M. Glass-Maujean, J. Breton and P. M. Guyon, Z. Phys. D5, 189 (1987).

13. D. R. Bates and J. T. Lewis, Pro. Phys. Soc. A68, 173 (1955).

14. H. A. Bethe and E. E. Salpeter, "Quantum Mechanics of One and Two Electron Atoms" (Springer, Berlin 1957). 